\title{
The Ascidian Fauna of the Plymouth Area.
}

\author{
By \\ N. J. Berrill, B.Sc., \\ Department of Physiology, University of Leeds.
}

THE nomenclature followed is that used by Hartmeyer (9), in which account will be found identificattion keys and complete lists of the synonymous species, only the commoner of which are given below. That his conclusions in this respect are open to question has already been pointed out (see Journ. Mar. Biol. Assoc., Vol. XV, p. 159).

For a description with figure of a species the reference number is placed after the name.

Those species common but widely scattered are denoted thus *, those common but local **.

Keys to localities: Aquarium tanks, at. Asia shoal, a. Bridge ground, $b$. Cawsand Bay, c. Duke Rock, d. Drake's Island, $d r$. Eddystone Grounds, $e$. Mewstone ledge, $f$. Millbay channel, $g$. Millbay Pier, $h$. New Grounds, $i$. Millbay Docks, $j$. Queen's Ground, $k$. Rame, $l$. RameEddystone, $m$. Stoke Point, $n$. Salcombe (Castle Rocks), o. Salcombe (Salstone), $p$. Shore below Laboratory, $q$. Skerries, $s$. Torbay, $t$. Yealm River (hotel corner), $y$. Wembury, $w$. For outer western area stations (Crawshay, 3), station number.

The months in which the different species are recorded to breed are denoted by Roman numerals, but the breeding season may in any case be of longer duration than that shown.

The records include those in the "Plymouth Invertebrate Fauna " (1), in the "Fauna of the Outer Western Area " (3), from collections in the museum of the Plymouth Laboratory identified (1911) by Hartmeyer, and those made by myself during the last four years.

\section{Molgulidæ,}

Eugrra arenosa Ald. (14). Deep water, free in sand or gravel. m.1.6.10.37.

Molgula manhattensis de Kay, var. ampulloides. = M. ampulloides Bened. (14). Low water, fixed. I-XII. j.p.y.

**Molgula oculata Forb. (14). Deep water, free in sand or gravel. VII-IX. c.m.11.33.46. 
**Molgula occulta Kupfif. =M. roscovitc Lac.-Duth. (14). ? $+M$ conchilega Forb. In association with $M$. oculata. VII-IX. c.m. 11.33.46.

Molgula simplex Ald. (14). Deep water, fixed. V-VIII. eith.1.3.7. 8.10.11.12.31.32.34.49.70.

Molgula complanata Ald. (14). Shallow water and intertidal, fixed. VII-IX. a.o.

\section{Tethyidæ.}

Pyura tessellata Forb. (15). =Forbesella tessellata Lac.-Duth. Shallow to deep water. VIII. e.f.n.p.

Pyura squamulosa Ald. (10). $f$.

Pyura savignyi Phil. (15). =Pyura (Cynthia) morus Forb. Shallow to deep water. VIII. f.m.p.s.3.4.8.15.34.43.

Microcosmus clandicans Sav. (15). t.f.

\section{Styelidæ.}

Polycarpa pomaria Sav. (15) ( $(\boldsymbol{g}) . \quad$ ?= P. varians + P. tuberosa Lac.-Duth. Deep water. VIII-IX. e.f.g.k.y.1.3.4.7.8.9.12.49.59.60.70.72.

Polycarpa fibrosa Stps. (15). = P. comata Ald. Shallow water. V-IX. k.p.y.1.4.10.31.53.

*Polycarpa Rustica Lac.-Duth. (15). Shallow water. V-IX. $p$.

Polycarpa gracilis Hell. = P. tenere Lac.-Duth. (15). Shallow water. $f . y$.

**Styelopsis (Dendrodoa) grossularia Bened. (15). Shallow water and intertidal. IV-X. a.f.h.q.y.

Stolonica socialis Hartmr. (15). = Thylacium aggregatum (Ald.). Deep water. VIII-IX. b.d.k.f.

*Distomus variolosus Gaertn. (15). = Heterocarpa glomerata Lac.-Duth. Shallow water and intertidal. VII-IX. a.c.f.k.

\section{Botryllidæ.}

*Botryllus schlosseri Pall., var. typica (6). Shallow water and intertidal. VI-VIII. g.k.l.m.o.q.

Botryllus SChlOSSERI, var. POLyCyClus (4). = B. gigas + Polycyclus polycyclus. Shallow to deep water. a.e.f.3.4.7.8.48.60.64.

*Botryllus leachiI Sav. (6). =Botrylloides vinosum+rubrum, etc., Milne-Edw. Shallow water and intertidal. VIII-X. i.q.p.3. 4.8.38.

\section{Rhodosamatidæ.}

Corella parallelogramma Müll. (y). =C. larvaformis Hanc. Deep water. VIII. b.d.e.g.f.3.10.32.38.60. 


\section{Ascidiidæ.}

*Ascidia mentula Müll. (4). Deep water. II-XI. f.g.h.n.p.3.4.12.34. 40.59 .

Ascidia conchilega Müll. (4). =A. depressa (Ald.). Low water to deep water. I-XII. h.e.g.p.1.4.8.10.32.34.36.37.43.49.70.

Ascidia virginea Müll. ( $\boldsymbol{y}) .=A$. venosa Traust. Deep water. VI-X. h.e.1.3.8.12.15.34.38.40.43.59.60.67.70.72.

**Ascidiella aspersa Müll. (12). Intertidal to deep water. I-XII. j.n.p.y.

*Ascidiella scabra Müll. (12). Shallow to deep water. V-IX. e.g.k.m.n.y.

* Phallusia mammillata Cuv. (18). Low water to deep water. I-XII. d.e.f.n.p.y.

\section{Cionidæ.}

*Ciona intestinalis Linn. (8). =C.fascicularis Hanc. Low water to deep water. IV-X. f.g.j.p.3.4.10.12.34.40.44.49.59.67.70.

Diazona violacea Sav. (4). Deep water. e.f.n.

\section{Perophoridæ.}

Perophora listeri Forb. (4). Tidal pools and shallow water. VIII-X. a.d.k.o.p.q.y.

\section{Polycitoridæ.}

**Clavelina lepadiformis Müll. (4). Low water and shallow water. VI-X. dr.d.f.g.k.j.p.

Clantelina auriludens Garst. (4). = Pycnoclavella aurilucens Garst. Deep water. VIII-IX. f.n.

Archidistoma AgGregatum Garst. (5). Shallow water. VI. $d$.

Holozoa (Distaplia) rosea d. Valle. (15). Shallow to deep water. k.d.

\section{Synoicidæ.}

Sidnyum turbinatum Sav. (6). =Circalium concrescens Giard. Shallow water and intertidal. b.dr.d.g.o.w.o.

Sidnyum elegans Giard (6). =Fragarium elegans Giard. Shallow water. d.g.i.

Amaroucium punctum Giard (6). Shallow to deep water. b.f.k.j.n.

*Amaroucium nordmanni Milne-Edw. (6). Low water and shallow water. VI-IX. k.m.p.w.

Amaroucium densum Giard (6). f.t.

Aplidium pallidum Verrill. (6). = A. zostericola Giard $+A$. lacteum Huit. Kaas. +Amaroucium pall. Verrill. Shallow to deep water. d.l.i.k. 
*Morchellium argus Milne-Edw. (10). =Morchelloides alderi (Herd.). Intertidal and shallow water. VI-IX. a.b.d.g.h.i.j.o.p.y.

Polyclinum aurantium Milne-Edw. (6). $=$ P. sabulosum Giard + Glossoforum sabulosum Giard + Aurantium aurantium Lah. Shallow to deep water. k.l.i.

\section{Didemnidæ.}

*Diplosoma listerianum M.E., var. gelatinosum M.E. (16).

=Didemnum gelatinosum + Leptoclinum gelatinosum M.E. Shallow to deep water. at.a.g.i.h.n.1.3.4.5.9.

Trididemnum tenerum Verrill. ( $(\mathbf{7})$. =Didemnopsis variabile Hartmr. + Didemnum cereum Giard. Intertidal to deep water. d.f.h.i.m.n.o.y.

\section{Doliolidæ.}

Doliolum nationalis Borgert. (2). In tow-nettings, Aug., Sept., 1893. Abundant 1895. A few S. of Eddystone, Nov., 1904.

\section{Salpidæ.}

SAlpa fusiformis Cuv. (13). Aggregated form at surface, N. of Eddystone, Aug., 1901.

Salpa mucronata Forskal. (13). Large shoals in Sound, 1893, in June and July. VIII-X.

\section{Appendicularia.}

OIkopleura dioica Fol. (17). Tow-nettings. III-IV. Fritillaria Borealis $\left(\mathbf{1}^{\mathbf{y}}\right)$. Tow-nettings.

\section{REFERENCES.}

1. Allen, E. J. 1904. The Plymouth Marine Invertebrate Fauna. Journ. Mar. Biol. Ass., N.S., Vol. VII, No. 2.

2. Borgert, A. 1894. Ergbn. Plankton Exped., vol. 2, pt. E.a.C.

3. Crawshay, L. R. 1912. On the Fauna of the Outer Western Area of the English Channel. Journ. Mar. Biol. Ass., N.S., Vol. XII.

4. Garstang, W. 1891. Report on the Tunicata of Plymouth. Pt. 1. Journ. Mar. Biol. Ass., N.S., Vol. II.

5. Garstang, W. 1891. Note on a new and primitive type of Compound Ascidian. Ann. Mag. Nat. Hist., ser. 6, vol. 8.

6. Giard, A. 1872-3. Les Synascidies. Arch. Zool. expt., ser. 1, vol. 1 and 2. 
7. Hartmeyer, R. 1901. Meeresfauna, Bergen. (Bergen Mus. Publ.), pt. 1.

8. Hartmeyer, R. 1903. Fauna arctica, vol. 3.

9. Hartmeyer, R. 1923. Ascidiacea. Danish Ingolf Expedition.

10. Heller. 1877. Untersuchungen über die Tunicaten des Adriatischen und Mittelmeeres. III (1). Densk. Ak. Wien., vol. 37.

11. Herdman, W. 1891. A Revised Classification of the Tunicata. Pt. 1, Journ. Linn. Soc., Zool. vol. 23.

12. Herdman, W. 1893. Pt. 2. Ibid., vol. 24.

13. Herdman, W. 1873-6. Report Voyage Challenger, vol. 27.

14. LaCaze-Duthiers, H. DE. 1877. Les ascidies simples des côtes de France. Arch. Zool. expt., ser. 1, vol. 6.

15. Lacaze-Duthiers, H. de, and Delage, Y. 1892. Faune de Cynthiadées. Mem. pres. Ac. France, vol. 45.

16. Lahille. 1890. Recherches Tunicates. Toulouse.

17. Lohmann, H. 1896. Ergbn. Plankton Exped., vol. 2, pt. E.c.

18. Traustedt, M. P. A. 1883. Mitth. Zool. Stat. Neapel., vol. 4. 
